\title{
Mn-containing Titanium surface with favorable osteogenic and antimicrobial functions synthesized by PIII\&D
}

Le Yu $u^{\S}$, Yaxin Tian, Yuqin Qiao and Xuanyong Liu*

State Key Laboratory of High Performance Ceramics and Superfine Microstructure, Shanghai

Institute of Ceramics, Chinese Academy of Sciences, Shanghai 200050, China

${ }^{\S}$ Present address: Department of Materials Science \& Engineering, University of Connecticut, Storrs, CT 06269, USA

\section{* Corresponding Author}

Prof. Xuanyong Liu

State Key Laboratory of High Performance Ceramics and Superfine Microstructure, Shanghai Institute of Ceramics, Chinese Academy of Sciences, Shanghai 200050, China

E-mail: xyliu@mail.sic.ac.cn

Tel: 86-21-52412409 


\begin{abstract}
Reasonable incorporation of manganese into titanium is believed to be able to enhance the osteogenic and antibacterial activities of orthopedic implants. However, it is still a challenge to compromise Mn-induced cytotoxicity and better develop its biocompatibility and antimicrobial ability. To pinpoint this issue, a stable $\mathrm{Mn}$ ion release platform was created on $\mathrm{Ti}$ using plasma immersion ion implantation and deposition (PIII\&D) technique. Compared with as-etched titanium, as a result, promoted antibacterial abilities against gram-negative bacteria species and enhanced osteogenic-related gene expressions on rBMMSC were observed on Mn-containing sample. Meanwhile, the Mn-containing samples showed no obvious cytotoxicity. Our results here provide insight to be better understanding the relationships between additives-induced biological performance and the dose, state, and stability of the doped element.
\end{abstract}

Keywords: manganese, titanium, PIII\&D, antibacterial, osteogenesis 


\section{Introduction}

It is popularly accepted that the interactions between implant surface and host cells have a significant effect on determining the efficiency of surgical implant operation [1]. Increasing research has focused on surface modification of the most wildly used Ti-based orthopedic materials [2,3]. Among which, our group has paid considerable attention on the surface loading of inorganic additives such as $\mathrm{Mn}$ [4], $\mathrm{Fe}$ [5], $\mathrm{Cu}[6]$, $\mathrm{Zn}[7,8], \mathrm{Ag}[9]$, or combined dual elements $[10,11]$ on Ti to promote the osteogenic ability and better compromise the cytotoxicity and antibacterial activities of the additives using various techniques. One suggestion in common from these researches is that the biological response to material depends more or less on the concentration, existence form, and stability of the additives.

Particularly, the coatings with reasonable incorporation of Mn that synthesized through Micro Arc Oxidation (MAO) technique on Ti showed good biological performance overall. Whereas adverse rat bone marrow mesenchymal stem cells (rBMMSC) proliferation rate and ALP expression were still observed from the surface containing relatively high dosage of $\mathrm{Mn}$ [4]. In addition, there are plenty researches verified that biomaterials overdosed by $\mathrm{Mn}$ could be detrimental to cells. Luthen et al. [12] revealed obvious cytotoxicity on human osteoblast of high dosage $\mathrm{MnCl}_{2}$-containing culture medium, while no obvious adverse effect can be detected from the relatively lower one. Park et al. [13] investigated Mn-induced cytotoxicity on nanostructured Mn-incorporated Ti oxide layer with $1.65 \mathrm{mg} / \mathrm{L} \mathrm{Mn}$ ions released within first $24 \mathrm{~h}$. Stredrick et al. [14] demonstrated that the Mn concentrations 
resulted in a half viability $\left(\mathrm{EC}_{50}\right)$ of CATH.a cells and SK-N-SH cells are $3.3 \mathrm{mg} / \mathrm{L}$ and $11.0 \mathrm{mg} / \mathrm{L}$ respectively.

There was a case compared the biological behaviors of bulk-incorporated Zn-containing $\mathrm{Ti}$ synthesized by MAO technique and subsurface-incorporated Zn-containing $\mathrm{Ti}$ obtained via PIII\&D method. Up-regulated in vitro osteogenic-related gene expressions and improved in vivo bone growth stimulation were detected from the subsurface-implanted $\mathrm{Zn}$-containing $\mathrm{Ti}$ compared with the other [15]. It enlightens us on implanting Mn onto the very subsurface of Ti using PIII\&D to stabilize the additive and further reduce the ion release ratio, thus to yield better biological benefit of manganese without introducing any Mn-induced cytotoxicity.

PIII\&D is a versatile technique, which is capable of modifying its surface characteristics without changing the bulk properties of substrates, and allows yielding suitable surface chemical composition through predetermining the metallic cathode and bias parameters [16]. Herein, we fabricated and characterized two groups of Mncontaining titanium using PIII\&D technique. The in vitro antimicrobial behaviors against gram-negative Pseudomonas aeruginosa (P. aeruginosa) and Escherichia coli (E. coli), and osteogenic activities responded to rBMMSC of Mn-PIII\&D samples compared with as-etched $\mathrm{Ti}$, were investigated.

\section{Materials and methods}

\subsection{Mn-PIII\&D}


Commercial pure Ti plates (Cp Ti, Grade 2) with dimensions of $10 \times 10 \times 1 \mathrm{~mm}^{3}$, or $20 \times 20 \times 1 \mathrm{~mm}^{3}$ were pretreated by etching against a mixed acid solution $\left(\mathrm{HF}: \mathrm{HNO}_{3}\right.$ : $\mathrm{H}_{2} \mathrm{O}=1: 5$ : 34) in sonicator for 10 min twice, followed by sonicating against ethanol and deionized water for several times, and then dried in room temperature for further use (as-etched Ti). Mn-containing modification layer were fabricated on as-etched Ti by PIII\&D equipment (PIIIS-700, Southwest Institute of Nuclear Industry, China) with pure manganese cathode. To avoid burst transport during PIII\&D process, recirculation cooling water system was applied to help the temperature around sample stage maintains to be $30 \sim 40{ }^{\circ} \mathrm{C}$. In addition, a magnetic duct with a curved shape was inserted between the plasma source and main chamber in our instrument to remove macro-particles produced from the cathodic arc. The key processing parameters of Mn-PIII\&D are listed in Table 1. The samples prepared with voltage pulse duration of $500 \mu$ s were labeled as Mn5, and the samples prepared with that of $800 \mu$ s were labeled as Mn8.

\subsection{Surface characterization and manganese release}

The surface topographies of various samples were observed by field emission scanning electron microscopy (FESEM; Magellan-400, FEI, USA). The surface and interior layer elemental compositions, binding states and manganese depth profiles of various specimens were investigated by X-ray photoelectron spectrometry (XPS; Physical Electronics PHI 5802, USA) with a monochromatic Al Ka source. The manganese-containing samples were soaked in $5 \mathrm{~mL}$ of physiological saline solution 
at $37{ }^{\circ} \mathrm{C}$ without stirring for 2 weeks. At each time point, the leaching liquid was collected and the concentration of Mn ions released into solution was analyzed by inductively coupled plasma optical emission spectrometry (ICP-OES).

\section{3. $\mathrm{rBMMSC}$ responses}

\subsubsection{Cell culture}

The cytocompatibility of various samples to rBMMSC (Stem Cell Bank, Chinese Academy of Sciences, China) was determined by investigating the cell attachment, proliferation, alkaline phosphate (ALP) activity, collagen secretion and osteogenic-related gene expressions. In a humidified incubator (STIK, China) that maintains constant temperature at $37{ }^{\circ} \mathrm{C}$ and supplied with $5 \% \mathrm{CO}_{2}$, cells were cultured in a petri dish (Thermo Scientific, USA) containing $8 \mathrm{ml}$ of $\alpha$-minimum essential medium ( $\alpha$-MEM; Gibco, Invitrogen, Inc, USA), which was supplemented with $10 \%$ fetal bovine serum (FBS; Gibco, Invitrogen, Inc, USA) and $1 \%$ penicillin-streptomycin (Gibco, Invitrogen, Inc, USA). The culture medium was refreshed every 3 days. In vitro experiments were carried out between passages 3 and 6 of rBMMSC, and quantitative assays were conducted in triplicate.

\subsubsection{Cell attachment and viability}

The rBMMSC were seeded on various samples in 24-well plates at a density of $2 \times$ $10^{4}$ cells per well supplemented with $1 \mathrm{ml}$ culture medium to observe the spreading status of rBMMSC on various samples at different time point. After incubated for 1,4 , 
and 7 days respectively, samples attached with cells were fixed with $2.5 \%$ glutaraldehyde solution (Gibco, Invitrogen, USA) for $30 \mathrm{~min}$, followed by dehydrated against a series of ethanol solutions (30, 50, 75, 90, 95, and 100\% (v/v)) for $10 \mathrm{~min}$ each. After the final dehydration immersed in absolute ethanol twice, samples were primarily dried with a series of hexamethyldisilizane (HMDS) solution solved in ethanol $(33,50,67$, and $100 \%(v / v))$. The samples were observed via SEM (S-3400, Hitachi, Japan) after finally being air-dried.

Similarly, the rBMMSC were seeded on various samples with four replicates for each group in 24-well plates at a density of $2 \times 10^{4}$ cells per well and supplemented with $1 \mathrm{ml}$ of culture medium to observe the cell proliferation and viability according to alamarBlue (AbD Serotec Ltd, UK) assay. After being incubated for 1, 4, and 7 days, samples attached with cells were transferred to a new 24-well plate and $1 \mathrm{ml}$ of phenol red free fresh medium supplemented with 5\% alamarBlue was added to each well. After cultured for another $4 \mathrm{~h}, 100 \mu \mathrm{l}$ supernatant of culture medium from each well was transferred to a 96-well plate to measure the absorbance at $570 \mathrm{~nm}$ and 600 nm by a plate reader (MK3, Thermo Scientific, USA). Then the cell proliferation ratio was calculated following the protocol of the alamarBlue assay.

\subsubsection{ALP activity}

The ALP activity of rBMMSC seeded on Mn8 and as-etched Ti were measured. After being cultured on various specimens with size of $10 \times 10 \times 1 \mathrm{~mm}^{3}$ for 7 and 14 days in 24-well plates at a density of $1 \times 10^{4}$ cells per well and supplemented with $1 \mathrm{ml}$ 
culture medium, cells were transferred to a new 24 -well plate with samples. The cells were dissociated from samples with lysis buffer for $60 \mathrm{~min}$ at $4{ }^{\circ} \mathrm{C}$, and incubated with $100 \mu \mathrm{l}$ of $p$-nitrophenyl phosphate $(p N P P)$ and $20 \mu l$ of glycine buffer. The mixed solution was reacted for $30 \mathrm{~min}$ at $37^{\circ} \mathrm{C}$, and then quenched by adding $20 \mu \mathrm{l}$ of $1 \mathrm{M} \mathrm{NaOH}$. The ALP activity of rBMMSC was determined by measuring the absorbance at a wavelength of $405 \mathrm{~nm}$, while the total protein content was determined using a Bio-Rad protein assay kit (Bio-Rad, USA) at a wavelength of 570nm. The relative activity of ALP was normalized to the counterpart of total protein concentration.

\subsubsection{Collagen secretion}

The collagen secretion from the surface of Mn8 compared with as-etched Ti were measured on rBMMSC according to the methods reported in literature [17]. Briefly, after being cultured for 7 and 14 days respectively, samples attached with cells were washed twice against PBS, fixed with $4 \%$ paraformaldehyde for $20 \mathrm{~min}$ and stained for collagen secretion with $0.1 \mathrm{wt} \%$ sirius red that saturated in picric acid for $20 \mathrm{~h}$. Afterwards, cells were washed against $0.1 \mathrm{M}$ acetic acid for multiple times and images were taken under brightness field. To be quantitative, the stain on the specimens was dissolved in $500 \mathrm{ml}$ of de-stain solution (0.2 $\mathrm{M} \mathrm{NaOH}$ : methanol=1:1) and then the absorbance of solution at $492 \mathrm{~nm}$ was measured on plate reader.

\subsubsection{Real-time quantitative PCR (RT-qPCR) analysis}


To investigate Mn-induced osteogenic activity on rBMMSC from gene level, osteogenic-related gene expressions including alkaline phosphatase (ALP), bone morphogenetic protein 2 (BMP-2), osteopontin (OPN), and runt-related transcription factor 2 (Runx2) were analyzed on Mn8 and Ti with $\beta$-actin as the housekeeping gene for normalization and relatively quantified using RT-qPCR. The rBMMSC at density of $1 \times 10^{5}$ cell/well were introduced on three identical specimens with dimension of $20 \times 20 \times 1 \mathrm{~mm}^{3}$ in a petri dish for 7 , and 14 days, respectively. At the end of each incubation point, samples attached with cells were washed twice against PBS and the total RNA was extracted by $1 \mathrm{ml}$ of the TRIzol reagent (Invitrogen, USA). The complementary DNA (cDNA) was reverse -transcribed from $1 \mathrm{mg}$ of total RNA using a Transcriptor First Strand cDNA Synthesis Kit. Afterwards, RT-qPCR was executed on the Roche LightCycler480 system (Roche, Switzerland) using an SYBR Green I PCR Kit. The relative expressions for the target genes were normalized to that of the reference gene $\beta$-actin and the primers for RT-PCR are listed in Table 2.

\subsection{Antibacterial ability}

The antibacterial activity of sample that containing relative higher dose of Mn (Mn8) compared with as-etched Ti against gram-negative both $E$. coli and $P$. aeruginosa was investigated by statistical investigations, including bacterial counting method and alamarBlue assay, and further confirmed via SEM observation.

Specimens with size of $10 \times 10 \times 1 \mathrm{~mm}^{3}$ were sterilized against $75 \%$ Ethanol for 4 times (30 min each), followed by air dried in hood. Then samples were transferred 
to a new 24-well culture plate. A drop of $60 \mu \mathrm{l}$ bacteria solution at a density of $10^{7}$ $\mathrm{cfu} / \mathrm{ml}$ was introduced on each sample. After incubated at $37{ }^{\circ} \mathrm{C}$ in dark for $24 \mathrm{~h}$, samples were rinsed with physiological saline solution twice. Then each sample and attached bacteria solution was transferred into a tube containing $4 \mathrm{ml}$ of physiological saline. The tubes were vigorously shaken for $60 \mathrm{~s}$ using a vortex mixer to dissociate bacteria from sample surfaces. Subsequently, the detached bacterial suspension was diluted in 10 -fold step by step with sterile physiological saline. $100 \mu$ of diluted bacterial solution was re-cultured on a standard Luria-Bertani (LB) agar gel culture medium at $37{ }^{\circ} \mathrm{C}$ in dark for another $16 \mathrm{~h}$. Then the active bacterial colonies were counted according to the National Standard of China GB/T 4789.2 protocol and the antibacterial ratio was calculated as follows,

$$
\frac{(A-B)}{A} \times 100 \%
$$

in which, A is the average number of bacterial colonies detached from as-etched $\mathrm{Cp}$ $\mathrm{Ti}$ (CFU/sample); $\mathrm{B}$ is the total number of bacterial colonies detached from Mn-containing sample (CFU/sample).

The samples for alamarBlue assay and SEM observation were obtained following the procedures described in section 2.3.2 on rBMMSC; the bacterial concentration seeding for alamarBlue assay was $10^{7} \mathrm{cfu} / \mathrm{ml}$.

\subsection{Statistical analysis}

Statistically significant differences $(\mathrm{P})$ between various groups were measured using one-way analysis of variance or Tukey's multiple comparison tests that executed 
through GraphPad Prism 5.

\section{Results and discussions}

\subsection{Surface characterization and Mn release}

As shown in Fig. 1a-c, the surface morphologies of as-etched Ti and Mn-containing samples have no obvious difference; all showing gully-like structures that arose from the pretreatment of acid etching. In addition, it can be found that numerous nano-grains with diameters between 5 20 $\mathrm{nm}$ appear on the surface of as-etched $\mathrm{Ti}$ (Fig. 1d), which was reported as obtained from pickling process as well $[6,18]$. While after Mn-PIII\&D, the nano-grains disappeared and the surfaces of Mn-containing samples (Fig. 1e and 1f) became smooth compared with as-etched Ti. It possibly results from the high energy bombardment of the Mn plasma that generated from cathode arc and accelerated by the high negative bias during Mn-PIII\&D [19].

Mn-PIII\&D samples were analyzed by XPS to assess the chemical states and Mn elemental distributions along with depth profile. Fig. 2a-d show the Mn 2p spectra obtained from the surface and interior layer of Mn-PIII\&D specimens. The Mn $2 p$ doublet obtained from the surfaces of Mn5 and Mn8 both located at $653.4 \mathrm{eV}$ and $641.2 \mathrm{eV}$ (Fig. 2a and 2b), corresponding with the binding energies of $\mathrm{Mn} 2 \mathrm{p}_{1 / 2}$ and Mn 2 $\mathrm{p}_{3 / 2}$ in $\mathrm{Mn}_{2} \mathrm{O}_{3}$ respectively [20, 21]. The Mn 2P spectrum that examined from 20 $\mathrm{nm}$ cleaned layer of Mn8 located around $649.5 \mathrm{eV}$ and $638.5 \mathrm{eV}$, indicating that the interior layer of Mn8 consists only of pure metallic Mn [22]. In addition to the same 
metallic Mn doublet compared with the interior spectrum of Mn 8 that located at $649.5 \mathrm{eV}$ and $638.5 \mathrm{eV}$, characteristic peaks that located at $653.7 \mathrm{eV}, 642.8 \mathrm{eV}$, and $641.2 \mathrm{eV}$, which all corresponded with the binding energies of $\mathrm{Mn}_{2} \mathrm{O}_{3}[21,23,24]$, were detected from that of Mn5.

As shown in Fig. 2e, the depth profiles between 15 and $100 \mathrm{~nm}$ of Mn element from Mn5 and Mn8 samples resemble Gaussian distribution, with the peak distribution of Mn all at 30-50 $\mathrm{nm}$ away from surface. The surface enrichment of $\mathrm{Mn}$ on Mn5 and Mn8 compared with those in interior layer about $15 \mathrm{~nm}$ deep arises from deposition during Mn-PIII\&D process, indicating a Mn containing thin film was formed on the surfaces of both Mn5 and Mn8 [11]. The deposition depths were all about $15 \mathrm{~nm}$. The surface and peak Mn concentration of Mn5 were 6.28 at\% and 10.00 at $\%$, respectively. In comparison, those of Mn8 were 13.43 at\% and 17.00 at $\%$, respectively.

To understand the role of Mn ion plays in controlling the biological effects on cells and bacteria, the ion release characteristics from each Mn-containing sample that soaked in physiological saline for 2 weeks were collected. As shown in Fig. 2f, small amount of Mn ions could be continuously detected from Mn-containing samples all the time. Specifically, the total amounts of Mn ions leached from Mn5 and Mn8 within first $24 \mathrm{~h}$, which were $0.0073 \mathrm{mg} / \mathrm{L}(\mathrm{ppm})$ and $0.01 \mathrm{mg} / \mathrm{L}$ respectively, are much lower than those from the Mn-doped Ti synthesized by MAO technique in our previous study that caused adverse ALP activity and from the nanostructured Mn-doping Ti that caused obvious in vitro disadvantages on cell, which were 0.48 
$\mathrm{mg} / \mathrm{L}$ and $1.65 \mathrm{mg} / \mathrm{L}$, respectively $[4,13]$.

\subsection{In vitro cytocompatibility}

Fig. 3 shows the cell attachment on each sample after being incubated for 4 days. Well-extended and homogenously distributed cell clusters with entangled filopodia and lamellipodia can be observed both from Mn-free and Mn-containing samples, indicating that no obvious cytotoxicity was introduce during Mn incorporation. To determine the cell viability and proliferation of rBMMSC that seeded on various samples culturing for different days, alamarBlue assay was applied and the result is shown in Fig. 4a. No statistic difference $(\mathrm{P}>0.05)$ can be seen among the three groups of surfaces within 7 days, further confirming that Mn-PIII\&D samples introduced no cytotoxicity to rBMMSC. In addition, the ALP activity of rBMMSC cultured on as-etched Ti and Mn8 that contained relatively higher Mn content for 7 and 14 days showed no statistic differences either (Fig. 4b; P>0.05). As a major marker that determines the quantity and quality of bones formed around the implant, collagen secretions of rBMMSC that cultured on Mn8 compared with Ti were measured. Fig. 4c shows that the collagen deposition of rBMMSC on Mn8 was statistically higher than that on $\mathrm{Ti}$ on day 14, while there was no detectable difference between these two groups on day 7. RT-qPCR analysis on osteogenesis-related gene expressions including ALP, BMP-2, OPN and Runx2 of rBMMSC seeded on Ti and Mn8 for 7 and 14 days are displayed in Fig. 4d-g, respectively. Significantly up-regulated gene expressions were detected on most of the investigated markers within 14 days and 
none showed any unfavorable effect.

In combination, Mn-PIII\&D samples showed no adverse effect on the proliferation and osteogenic differentiation of rBMMSC. Instead, Mn8 that contained relatively higher Mn content and be able to release more Mn ion imparts excellent osteogenic activity with enhanced collagen secretion and osteogenesis-related gene expressions of rBMMSC. It is popularly accepted that manganese plays key role in osteogenesis and osteoinduction activities of cells through interfering carbohydrate metabolism and mucopolysaccharides synthesis processes in bone $[25,26]$. In addition, numerous studies have verified that manganese deficiency in vivo could cause dysfunction of bone metabolism and bone growth [27, 28]. Therefore, we believe that the favorable effects on rBMMSC observed from Mn8 can be attributed to the presence of Mn that implanted in the supernatant of $\mathrm{Ti}$ and the subsequently gentle release of $\mathrm{Mn}$ ion, which was wildly accepted to be capable of altering the local micro-environment around biomaterials and consequently contributes to in vitro or in vivo cell functions $[29,30]$.

\subsection{Antibacterial activity}

Mn ion or Mn oxide has been verified to be able to inhibit bacterial proliferation or adhesion especially against gram-negative species in certain circumstances $[4,31,32]$. Therefore, in the present work, the bacterial morphology observation, alamarBlue assay, and the bacteria counting methods were carried out on Mn8 and Ti against $E$. coli and $P$. aeruginosa, respectively. As presented in Fig. 5a and b, the viabilities of 
both species assessed by alamarBlue on Mn8 were significantly lower than those on as-etched $\mathrm{Ti}$, verifying that $\mathrm{Mn}$ induced unfavorable effects against the proliferation of $E$. coli and $P$. aeruginosa. To quantify the antibacterial efficacies, the attached bacteria were detached and re-cultivated on agar plates according to the bacterial counting method. As shown in Fig. 5e, the total amounts of both kinds of colonies that detached from Mn8 and re-attached on agar plate were less than those from as-etched Ti. The statistical results are presented in Fig. $5 \mathrm{c}$ and d, showing that the antibacterial ratios against $E$. coli and $P$. aeruginosa of Mn8 compared with Ti were $20 \pm 6 \%$ and $\sim 58 \pm 4 \%$, respectively. The morphologies of bacteria seeded on various surfaces were observed via SEM and the results are shown in Fig. 6. The total amounts of both bacterial species attached on Mn8 and Ti showed no obvious differences. Whereas, it was found with high magnification SEM images that the cell membranes of E. coli and P. aeruginosa seeded on Mn8 (insets in Fig. 6c and d) all showed shriveled and deformed status. In comparison with the relatively intact and rod-like bacterial membranes that spread on the surface of Ti (insets in Fig. 6a and b), it is further confirmed that Mn8 sample was at least cytostatic to E. coli and P. aeruginosa. As mentioned in section 3.1, the Mn ion release rate within first $24 \mathrm{~h}$ from Mn8 is only $0.01 \mathrm{mg} / \mathrm{L}$, which is much less than the minimum concentration of $12.5 \mathrm{mg} / \mathrm{L}$ that caused antibacterial activity according to reference [31]. Therefore, Mn ion release itself in the present study seems like not sufficient to generate Mn-induced antimicrobial activity. One possible source that Mn8 reduced the viability of both gram-negative bacteria could attribute to the existence form of implanted $\mathrm{Mn}$ as 
$\mathrm{Mn}_{2} \mathrm{O}_{3}$, which was reported possessing catalytic activity and being able to generate ROS groups that could inhibit the germination of bacteria $[4,33]$.

\section{Conclusions}

Mn was successfully implanted onto the supernatant of as-etched Ti substrate using PIII\&D technique. Thus, a stable Mn release platform that contributed to the up-regulated biological performance Ti implants was created. On one hand, the Mn-PIII\&D sample that contains relatively higher Mn content and possesses faster Mn release rate showed increased collagen deposition and significantly enhanced osteogenesis-related gene expressions, without introducing any detrimental effect to rBMMSC. On the other hand, Mn-PIII\&D sample was able to decrease the viability of gram-negative E. coli and P. aeruginosa.

\section{Acknowledgements}

Financial support from the National Science Foundation for Distinguished Young Scholars of China (51525207), National Natural Science Foundation of China (31570973), Shanghai Committee of Science and Technology, China (15441904900, 14XD1403900) are acknowledged.

\section{Notes and references}

[1] X.Y. Liu, P.K. Chu and C.X. Ding, Surface modification of titanium, titanium alloys, and related materials for biomedical applications, Materials Science \& Engineering R-Reports, 47 (2004) 49-121.

[2] M. Geetha, A.K. Singh, R. Asokamani and A.K. Gogia, Ti based biomaterials, the ultimate choice for orthopaedic implants - A review, Progress in Materials Science, 54 (2009) 397-425. 
[3] M. Viceconti, R. Muccini, M. Bernakiewicz, M. Baleani and L. Cristofolini, Large-sliding contact elements accurately predict levels of bone-implant micromotion relevant to osseointegration, Journal of Biomechanics, 33 (2000) 1611-1618.

[4] L. Yu, S. Qian, Y. Qiao and X. Liu, Multifunctional Mn-containing titania coatings with enhanced corrosion resistance, osteogenesis and antibacterial activity, Journal of Materials Chemistry B, 2 (2014) 5397-5408.

[5] Y. Tian, H. Cao, Y. Qiao, F. Meng and X. Liu, Antibacterial activity and cytocompatibility of titanium oxide coating modified by iron ion implantation, Acta biomaterialia, 10 (2014) 4505-4517.

[6] L. Yu, G. Jin, L. Ouyang, D. Wang, Y. Qiao and X. Liu, Antibacterial activity, osteogenic and angiogenic behaviors of copper-bearing titanium synthesized by PIII\&D, Journal of Materials Chemistry B, 4 (2016) 1296-1309.

[7] G. Jin, H. Cao, Y. Qiao, F. Meng, H. Zhu and X. Liu, Osteogenic activity and antibacterial effect of zinc ion implanted titanium, Colloids and Surfaces B: Biointerfaces, 117 (2014) 158-165.

[8] H. Hu, W. Zhang, Y. Qiao, X. Jiang, X. Liu and C. Ding, Antibacterial activity and increased bone marrow stem cell functions of $\mathrm{Zn}$-incorporated TiO2 coatings on titanium, Acta Biomaterialia, 8 (2012) 904-915.

[9] H. Cao, X. Liu, F. Meng and P.K. Chu, Biological actions of silver nanoparticles embedded in titanium controlled by micro-galvanic effects, Biomaterials, 32 (2011) 693-705.

[10] G. Jin, H. Qin, H. Cao, Y. Qiao, Y. Zhao, X. Peng, X. Zhang, X. Liu and P.K. Chu, Zn/Ag micro-galvanic couples formed on titanium and osseointegration effects in the presence of $\mathrm{S}$. aureus, Biomaterials, 65 (2015) 22-31.

[11] G. Jin, H. Qin, H. Cao, S. Qian, Y. Zhao, X. Peng, X. Zhang, X. Liu and P.K. Chu, Synergistic effects of dual $\mathrm{Zn} / \mathrm{Ag}$ ion implantation in osteogenic activity and antibacterial ability of titanium, Biomaterials, 35 (2014) 7699-7713.

[12] F. Luthen, U. Bulnheim, P.D. Muller, J. Rychly, H. Jesswein and J.G. Nebe, Influence of manganese ions on cellular behavior of human osteoblasts in vitro, Biomolecular Engineering, 24 (2007) 531-536.

[13] J.-W. Park, Y.-J. Kim and J.-H. Jang, Surface characteristics and in vitro biocompatibility of a manganese-containing titanium oxide surface, Applied Surface Science, 258 (2011) 977-985.

[14] D.L. Stredrick, A.H. Stokes, T.J. Worst, W.M. Freeman, E.A. Johnson, L.H. Lash, M. Aschner and K.E. Vrana, Manganese-induced cytotoxicity in dopamine-producing cells, Neurotoxicology, 25 (2004) 543-553.

[15] Y. Qiao, W. Zhang, P. Tian, F. Meng, H. Zhu, X. Jiang, X. Liu and P.K. Chu, Stimulation of bone growth following zinc incorporation into biomaterials, Biomaterials, 35 (2014) 6882-6897.

[16] P.K. Chu, Applications of plasma-based technology to microelectronics and biomedical engineering, Surface and Coatings Technology, 203 (2009) 2793-2798.

[17] W. Wang, L. Zhao, K. Wu, Q. Ma, S. Mei, P.K. Chu, Q. Wang and Y. Zhang, The role of integrin-linked kinase/beta-catenin pathway in the enhanced MG63 differentiation by micro/nano-textured topography, Biomaterials, 34 (2013) 631-640.

[18] J. Li, W. Zhang, Y. Qiao, H. Zhu, X. Jiang, X. Liu and C. Ding, Chemically regulated bioactive ion delivery platform on a titanium surface for sustained controlled release, Journal of Materials Chemistry B, 2 (2014) 283.

[19] H. Wang, T. Lu, F. Meng, H. Zhu and X. Liu, Enhanced osteoblast responses to poly ether ether ketone surface modified by water plasma immersion ion implantation, Colloids and Surfaces B: 
Biointerfaces, 117 (2014) 89-97.

[20] G. Allen, S. Harris, J. Jutson and J. Dyke, A study of a number of mixed transition metal oxide spinels using X-ray photoelectron spectroscopy, Applied Surface Science, 37 (1989) 111-134.

[21] M. Oku, K. Hirokawa and S. Ikeda, X-ray photoelectron spectroscopy of manganese-oxygen systems, Journal of Electron Spectroscopy and Related Phenomena, 7 (1975) 465-473.

[22] C.J. Jenks, S.-L. Chang, J.W. Anderegg, P.A. Thiel and D.W. Lynch, Photoelectron spectra of an Al 70 Pd 21 Mn 9 quasicrystal and the cubic alloy Al 60 Pd 25 Mn 15, Physical Review B, 54 (1996) 6301.

[23] Y. Umezawa and C.N. Reilley, Effect of argon ion bombardment on metal complexes and oxides studied by x-ray photoelectron spectroscopy, Analytical Chemistry, 50 (1978) 1290-1295.

[24] B.J. Tan, K.J. Klabunde and P.M.A. Sherwood, XPS studies of solvated metal atom dispersed (SMAD) catalysts. Evidence for layered cobalt-manganese particles on alumina and silica, Journal of the American Chemical Society, 113 (1991) 855-861.

[25] Y.J. Bae and M.H. Kim, Manganese supplementation improves mineral density of the spine and femur and serum osteocalcin in rats, Biological trace element research, 124 (2008) 28-34.

[26] J. Aaseth, G. Boivin and O. Andersen, Osteoporosis and trace elements--an overview, Journal of trace elements in medicine and biology : organ of the Society for Minerals and Trace Elements, 26 (2012) 149-152.

[27] C. Paluszkiewicz, A. Ślósarczyk, D. Pijocha, M. Sitarz, M. Bućko, A. Zima, A. Chróścicka and M. Lewandowska-Szumieł, Synthesis, structural properties and thermal stability of Mn-doped hydroxyapatite, Journal of Molecular Structure, 976 (2010) 301-309.

[28] W. Zhaojun, W. Lin, W. Zhenyong, W. Jian and L. Ran, Effects of manganese deficiency on serum hormones and biochemical markers of bone metabolism in chicks, Journal of bone and mineral metabolism, 31 (2013) 285-292.

[29] A. Bigi, B. Bracci, F. Cuisinier, R. Elkaim, M. Fini, I. Mayer, I.N. Mihailescu, G. Socol, L. Sturba and P. Torricelli, Human osteoblast response to pulsed laser deposited calcium phosphate coatings, Biomaterials, 26 (2005) 2381-2389.

[30] E. Gyorgy, P. Toricelli, G. Socol, M. Iliescu, I. Mayer, I.N. Mihailescu, A. Bigi and J. Werckman, Biocompatible Mn2+-doped carbonated hydroxyapatite thin films grown by pulsed laser deposition, Journal of biomedical materials research. Part A, 71 (2004) 353-358.

[31] M.S. Hassan, T. Amna, D.R. Pandeya, A.M. Hamza, Y.Y. Bing, H.C. Kim and M.S. Khil, Controlled synthesis of $\mathrm{Mn}(2) \mathrm{O}(3)$ nanowires by hydrothermal method and their bactericidal and cytotoxic impact: a promising future material, Applied microbiology and biotechnology, 95 (2012) 213-222.

[32] G. Panthi, A. Yousef, N.A.M. Barakat, K. Abdelrazek Khalil, S. Akhter, Y. Ri Choi and H.Y. Kim, Mn2O3/TiO2 nanofibers with broad-spectrum antibiotics effect and photocatalytic activity for preliminary stage of water desalination, Ceramics International, 39 (2013) 2239-2246.

[33] H.-L. Su, C.-C. Chou, D.-J. Hung, S.-H. Lin, I. Pao, J.-H. Lin, F.-L. Huang, R.-X. Dong and J.-J. Lin, The disruption of bacterial membrane integrity through ROS generation induced by nanohybrids of silver and clay, Biomaterials, 30 (2009) 5979-5987. 


\section{Tables}

Table 1. Important instrumental parameters used in Mn-PIII\&D.

\begin{tabular}{lcc}
\hline & Target & Cathodic arc \\
\hline Pulsing frequency $(\mathrm{Hz})$ & 5 & 5 \\
Voltage pulse duration $(\mu \mathrm{s})$ & 500 & $500(\mathrm{Mn} 5) ; 800(\mathrm{Mn} 8)$ \\
Implantation voltage $(\mathrm{kV})$ & -30 & \\
Implantation time $(\mathrm{h})$ & 1 & \\
Pressure $(\mathrm{Pa})$ & $5 \times 10^{-3}$ & \\
\hline
\end{tabular}

Table 2. Primer sequences used in RT-qPCR.

\begin{tabular}{lll}
\hline Gene (rat) & Primer sequences (F, forward; R, reverse; 5'-3') & Product size (bp) \\
\hline$\beta$-actin & F: CACCCGCGAGTACAACCTTC & 207 \\
& R: CCCATACCCACCATCACACC & \\
ALP & F: CGTCTCCATGGTGGATTATGCT & 209 \\
& R: CCCAGGCACAGTGGTCAAG & \\
BMP-2 & F: ATGGGTTTGTGGTGGAAGTG & 167 \\
& R: TGTTTGTGGAGTGGATGTC & \\
OPN & F: CAAGCGTGGAAACACACAGCC & 165 \\
& R: GGCTTTGGAACTCGCCTGACTG & \\
Runx2 & F: TCTTCCCAAAGCCAGAGCG & 154 \\
& R: TGCCATTCGAGGTGGTCG & \\
\hline
\end{tabular}




\section{Figure captions}

Fig. 1. Surface morphologies of various samples under low $(a \sim c)$ and high $(d \sim f)$ magnification detected by FE-SEM.

Fig. 2. Mn $2 \mathrm{p}$ high-resolution spectrum obtained from the surface $(\mathrm{a} / \mathrm{b})$ and the interior in $20 \mathrm{~nm}$ depth (c/d) of various samples detected by XPS; Mn depth profiles of various samples detected by XPS (e); and Mn release characteristics soaked in 0.9 wt $\% \mathrm{NaCl}$ solution (f). (error bars represent mean $\pm \mathrm{SD} ; \mathrm{n}=3$ )

Fig. 3. SEM morphologies of rBMMSC cultured on various samples for 4 days.

Fig. 4. Cell responses on Mn-PIII\&D and Ti samples: (a) alamarBlue reduction of rBMMSC cultured for 1,4 and 7 days; (b) Relative ALP activity of rBMMSC seeded on Mn8 and Ti for 7 and 14 days; (c) collagen secretion of rBMMSC cultured for 7 and 14 days; osteogenesis-related gene expressions of rBMMSC cultured for 7 and 14 days: (d) ALP, (e) BMP-2, (f) OPN, and (g) Runx2. (error bars represent mean \pm SD; $\mathrm{n}=3 ; * \mathrm{P}<0.05, * * \mathrm{P}<0.01, * * * \mathrm{P}<0.001)$

Fig. 5. Antibacterial activity of Mn-PIII\&D and Ti samples: alamarBlue reductions of E. coli (a) and P. aeruginosa (b) cultured for $24 \mathrm{~h}$; percent reductions of bacteria re-cultivated on agar: E. coli (c) and $P$. aeruginosa (d) colonies were previously 
dissociated from various samples; (e) re-cultivated E. coli and P. aeruginosa colonies on agar for $16 \mathrm{~h}$ : bacterial colonies were previously dissociated from Mn8 and Ti that cultured for $24 \mathrm{~h}$ in dark. (error bars represent mean $\pm \mathrm{SD} ; \mathrm{n}=3 ; * \mathrm{P}<0.05, * * \mathrm{P}<0.01$, $* * * \mathrm{P}<0.001)$

Fig. 6 SEM morphologies of E. coli $(\mathrm{a} / \mathrm{c})$ and $P$. aeruginosa $(\mathrm{b} / \mathrm{d})$ cultivated on Mn8 and Ti for $24 \mathrm{~h}$ in dark. 


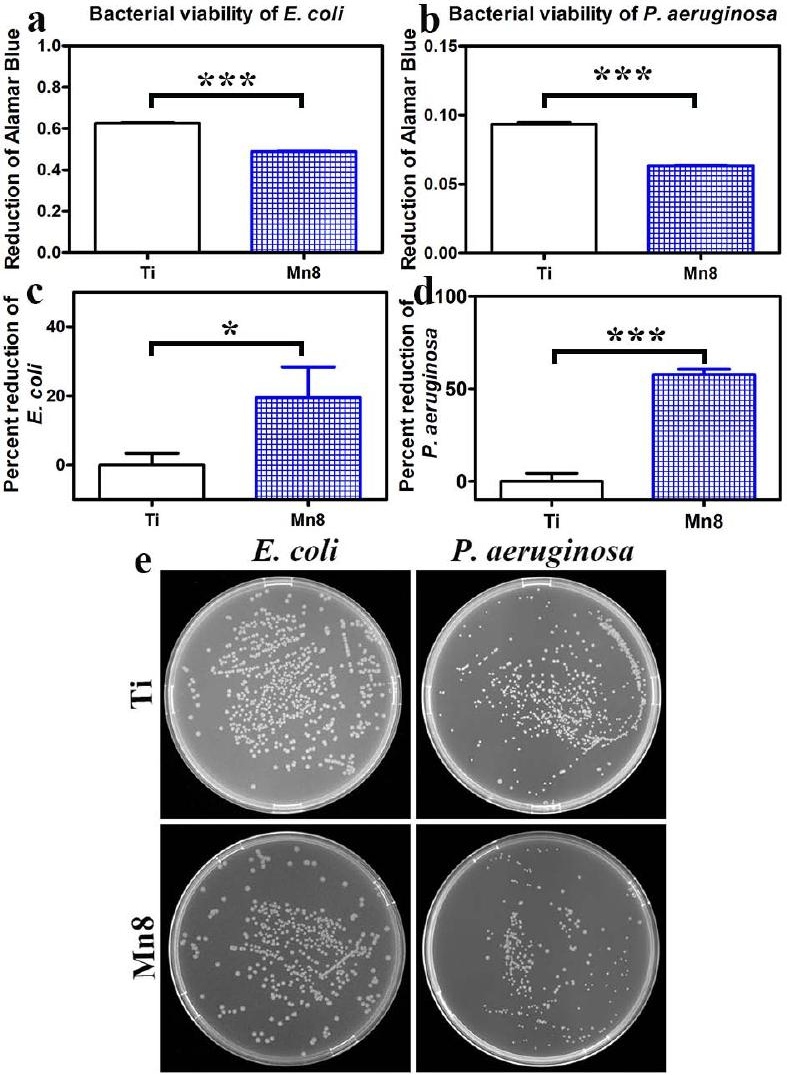


Ti

Mn5

Mn8

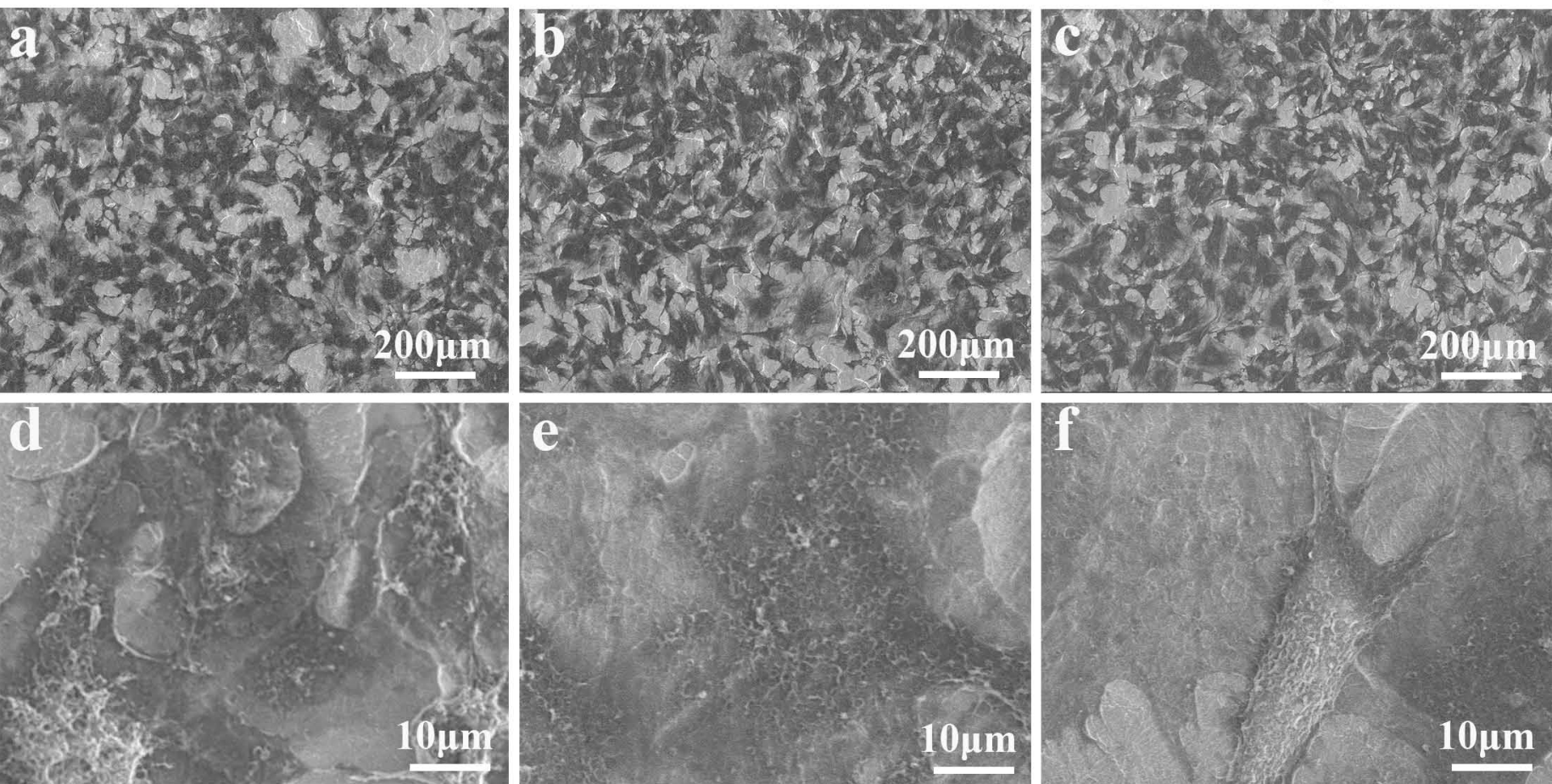




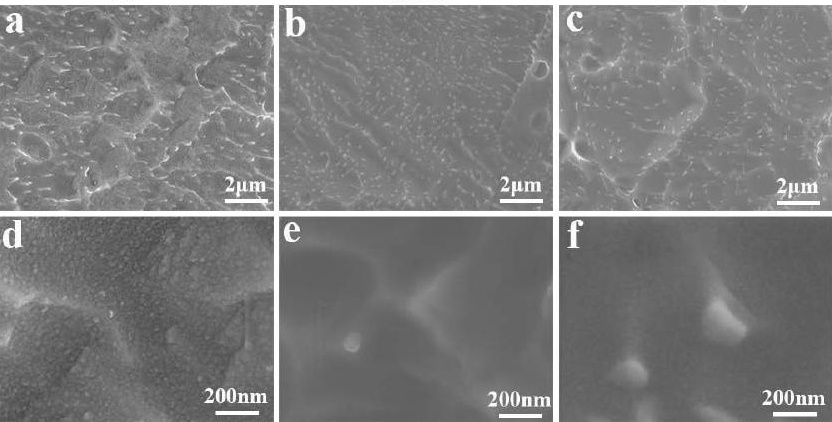


A stable $\mathrm{Mn}$ ion release platform was created on Ti by PIII\&D technique, and enhanced osteogenic and antibacterial abilities were observed.
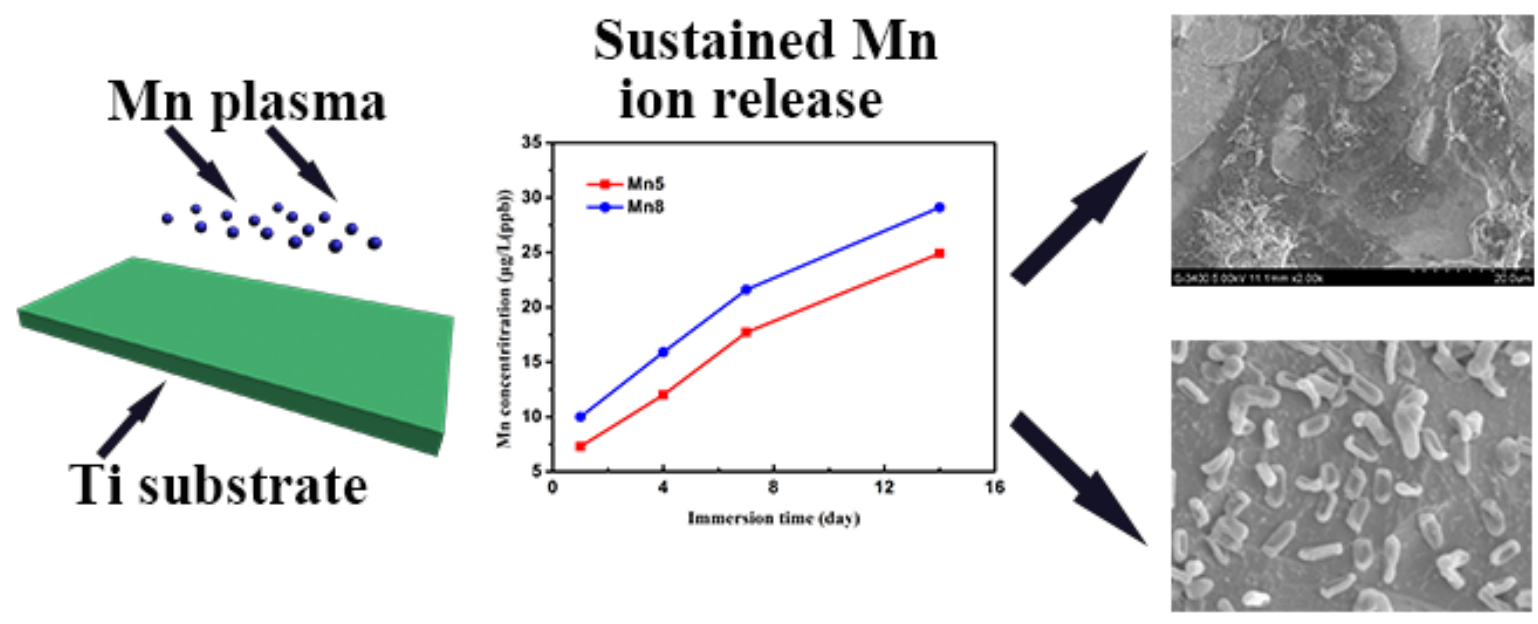